\title{
Large Deformation Analysis for Flexure Hinged Displacement Amplifier based on Minimum Potential Energy
}

\author{
Kang ZhangYi, Cheng Yong, Yu YueLing and Hu XiaoYin* \\ Shanghai Institute of Measurement And Testing Technology(SIMT), Shanghai, China, \\ Large Force Building 205, ZhangHeng Road 1500 \\ kangzy@simt.com.cn, chengy@simt.com.cn, yuyl@simt.com.cn, huxy@simt.com.cn
}

Keywords: Flexure hinge; Displacement amplifier, Large deformation analysis

Abstract. This paper presents a method for large deformation analysis for flexure hinged displacement amplifier by minimum potential energy. The flexure hinged displacement amplifier is widely used in micro electromechanical system, micro manipulation, gyroscopes. Different from conventional hinge, monolithic flexure hinge has the advantages of no friction, no wear, no backlash. With the great development, flexure hinge is not just applied in the elastic stage. But the nonlinear analysis of large deformation restricts the application of flexure hinge. This paper presents a method for large deformation analysis for flexure hinged displacement. The difference between small deformation and large deformation is discussed. And then a bridge-type displacement amplifier is designed and its performance is studied by MPE. The results by MPE and FEA is compared.

\section{Introduction}

To flexure hinge, there are no definite definitions to distinguish large and small deformation. The relationship between deformation and displacement can be expressed as Eq. 1 [1]:

$$
\left\{\begin{aligned}
\varepsilon_{x}= & \partial u_{x} / \partial x+1 / 2\left[\left(\partial u_{x} / \partial x\right)^{2}+\left(\partial u_{y} / \partial x\right)^{2}+\left(\partial u_{z} / \partial x\right)^{2}\right] \\
\varepsilon_{y}= & \partial u_{y} / \partial y+1 / 2\left[\left(\partial u_{x} / \partial y\right)^{2}+\left(\partial u_{y} / \partial y\right)^{2}+\left(\partial u_{z} / \partial y\right)^{2}\right] \\
\varepsilon_{z}= & \partial u_{z} / \partial z+1 / 2\left[\left(\partial u_{x} / \partial z\right)^{2}+\left(\partial u_{y} / \partial z\right)^{2}+\left(\partial u_{z} / \partial z\right)^{2}\right] \\
\gamma_{x y}= & 1 / 2\left(\partial u_{x} / \partial y+\partial u_{y} / \partial x+\partial u_{x} / \partial x \partial u_{x} / \partial y+\right. \\
& \left.\partial u_{y} / \partial x \partial u_{y} / \partial y+\partial u_{z} / \partial x \partial u_{z} / \partial y\right) \\
\gamma_{y z}= & 1 / 2\left(\partial u_{y} / \partial z+\partial u_{z} / \partial y+\partial u_{x} / \partial x \partial u_{x} / \partial z+\right. \\
& \left.\partial u_{y} / \partial x \partial u_{y} / \partial z+\partial u_{z} / \partial x \partial u_{z} / \partial z\right) \\
\gamma_{z x}= & 1 / 2\left(\partial u_{z} / \partial x+\partial u_{x} / \partial z+\partial u_{x} / \partial y \partial u_{x} / \partial z+\right. \\
& \left.\partial u_{y} / \partial y \partial u_{y} / \partial z+\partial u_{z} / \partial y \partial u_{z} / \partial z\right)
\end{aligned}\right.
$$

When the deformation is in the elastic stage, the formula (1) can be simplified as Eq. 2 [2]:

$$
\begin{aligned}
& \varepsilon_{x}=\partial u_{x} / \partial x \\
& \varepsilon_{y}=\partial u_{y} / \partial y \\
& \varepsilon_{z}=\partial u_{z} / \partial z \\
& \gamma_{x y}=\partial u_{x} / \partial y+\partial u_{y} / \partial x \\
& \gamma_{y z}=\partial u_{y} / \partial z+\partial u_{z} / \partial y \\
& \gamma_{z x}=\partial u_{z} / \partial x+\partial u_{x} / \partial z
\end{aligned}
$$




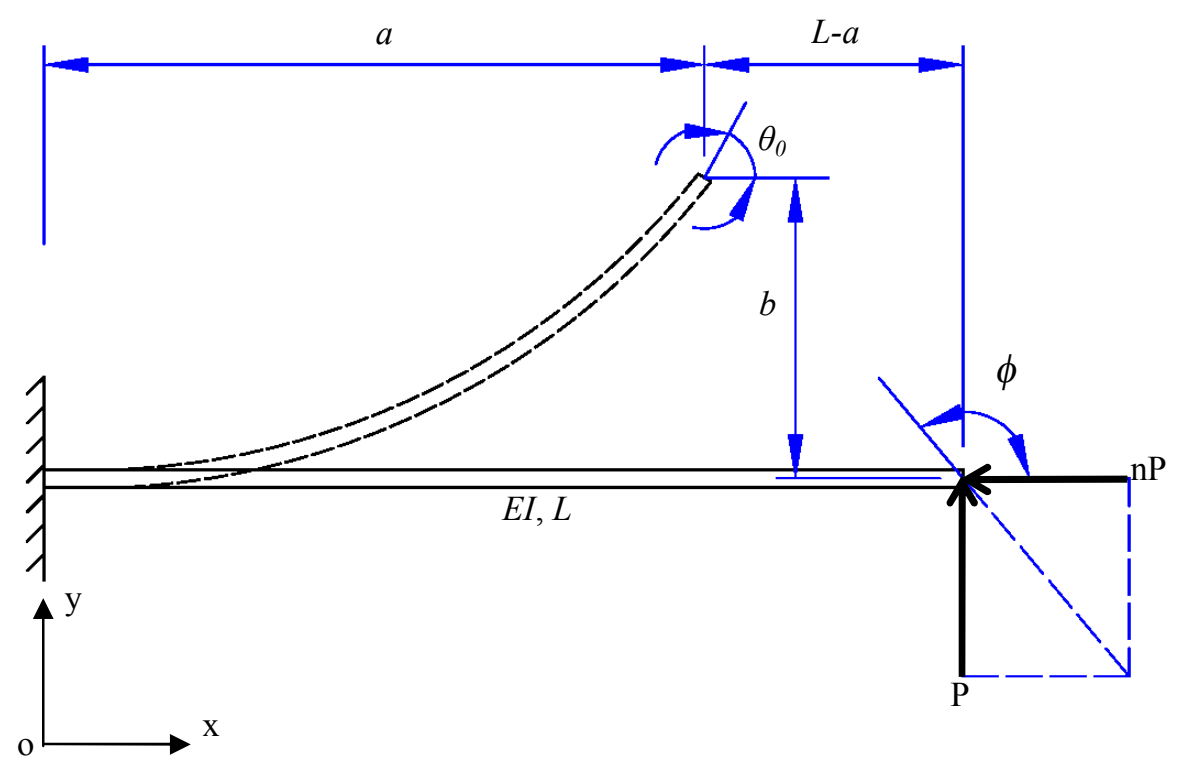

Fig. 1 End-force cantilever beam

Fig. 1 is a cantilever with a force in one end. Its relationship between deformation and displacement can be expressed according to Bemoulli-Euler formula as:

$M=E I \frac{d \theta}{d s}$

Where $M$ is toruqe, $d \theta / d s$ is curvature, $I$ is inertia moment. The curvature can be changed into:

$\frac{d \theta}{d s}=\frac{d^{2} y / d^{2} x}{\left[1+(\mathrm{dy} / \mathrm{dx})^{2}\right]^{3 / 2}}$

If the deformation is linear, $d y / d x$ can be ignored, and then

$M=E I \frac{d^{2} y}{d x^{2}}$

If the deformation is nonlinear, the curvature is:

$\frac{d \theta}{d s}=C \frac{d^{2} y}{d x^{2}}$

In which:

$C=\frac{1}{\left[1+(\mathrm{dy} / \mathrm{dx})^{2}\right]^{3 / 2}}$

To solve the problem of nonliear deformation analysis, FEA is the mostly used way. Reddy presented a automated synthesis procedure to design contact-aided compliant mechanism[3]. Hao designed multi-beam modules for the nonlinear analysis of spatial compliant parallel modules [4]. Aten applied shell and solid elements for nonlinear analysis of thin-film compliant MEMS [5]. The method of FEA has many advantages, but it's time-consuming, complicated and sometimes imprecise.

In this paper, the method of MPE is proposed and the analysis example is given. The analysis results are compared with that by FEA.

\section{MPE Analysis for a Displacement Amplifier}

The working principle of MPE is that the system reach the equilibrium state when its potential energy is minimum. It means the deformation or displacement of the system tends to reach the balance state. The objective function and constraints can be expressed as Eq. 8: 
Object function $\sum \frac{1}{2} k_{i}\left(\mathrm{x}_{i}-\mathrm{x}_{i 0}\right)^{2}$

$\sum_{i=1}^{n} \overrightarrow{x_{i}}=0$

$\sum_{i=1}^{n} \overrightarrow{y_{i}}=0$

$\sum_{i=1}^{n} \overrightarrow{z_{i}}=0$

Constraints

$$
\begin{aligned}
& \sum_{i=1}^{n} F_{x i}=0 \\
& \sum_{i=1}^{n} F_{y i}=0 \\
& \sum_{i=1}^{n} F_{z i}=0
\end{aligned}
$$

To verify the MPE method, a bridge-type displacement amplifier is designed as Fig. 2. According to the PRBM [5] (pseudo rigid model), the amplifier can be changed into a four DOFs (degree of freedom) model as shown in Fig. 3. The geometric parameter of flexure hinge and material parameter is listed in Table. 1. The different value of $h$ and $p$ of displacement amplifier are listed in Table. 2

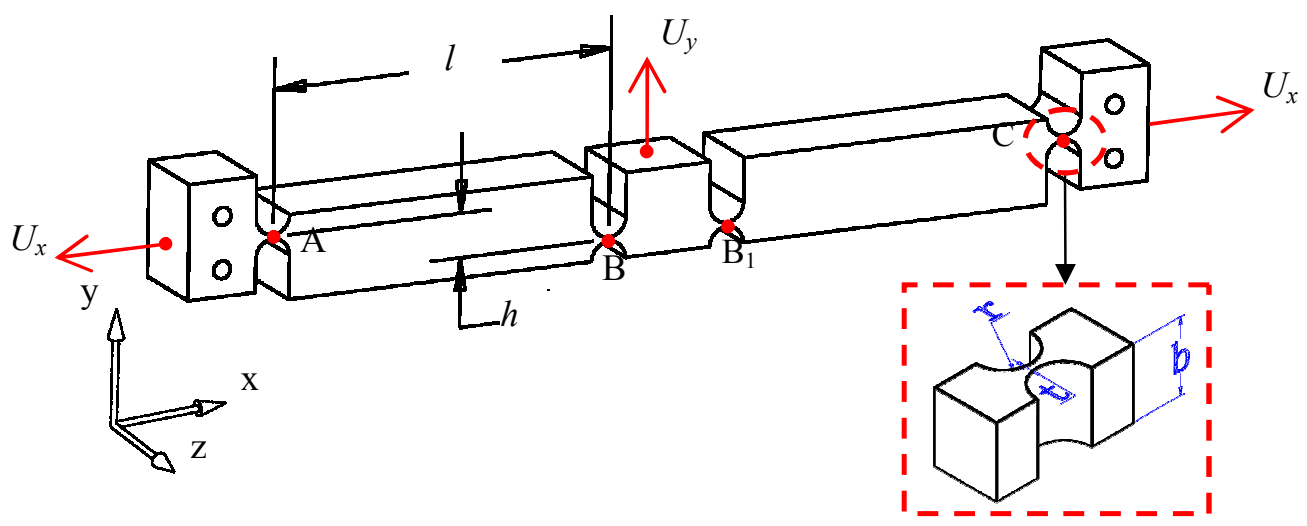

Fig. 2 Designed bridge-type displacement amplifier

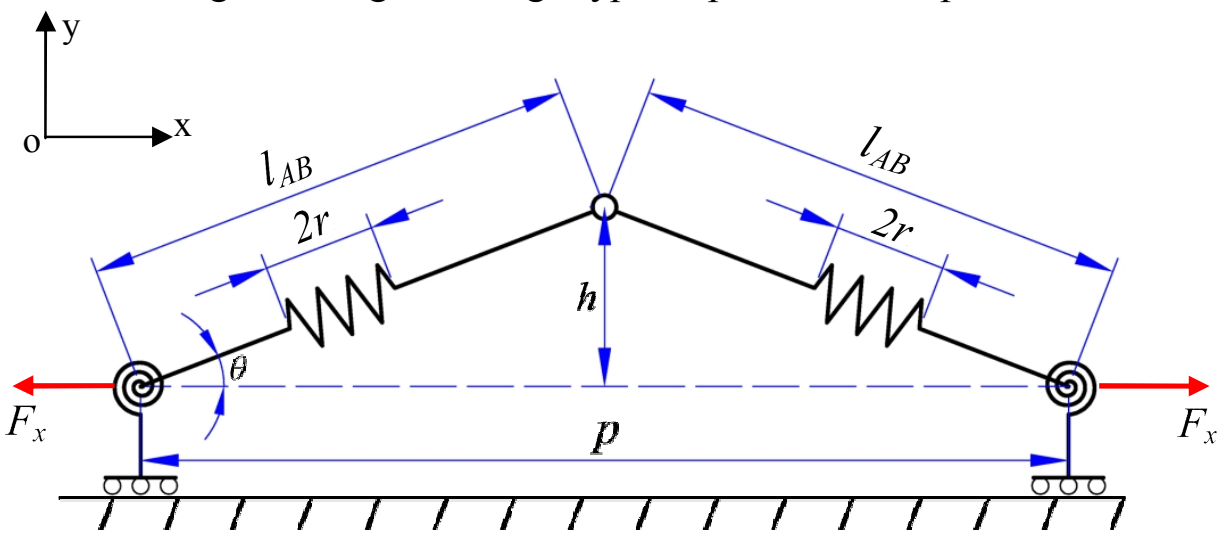

Fig. 3 Four DOFs model of the displacement amplifier 
Table. 1 Geometric and material parameter

\begin{tabular}{cc}
\hline Parameter & Value \\
\hline$b$ & $6 \mathrm{~mm}$ \\
$r$ & $2 \mathrm{~mm}$ \\
$t$ & $0.6 \mathrm{~mm}$ \\
$\mathrm{E}$ & 71.7 \\
$\gamma$ & 0.33 \\
\hline
\end{tabular}

Table. 2 Geometrical parameter of displacement amplifier

\begin{tabular}{ccc} 
No & $l(\mathrm{~mm})$ & $h(\mathrm{~mm})$ \\
\hline 1 & 40 & 5 \\
2 & 35 & 5 \\
3 & 30 & 5 \\
4 & 40 & 4 \\
5 & 35 & 4 \\
6 & 30 & 4 \\
\hline
\end{tabular}

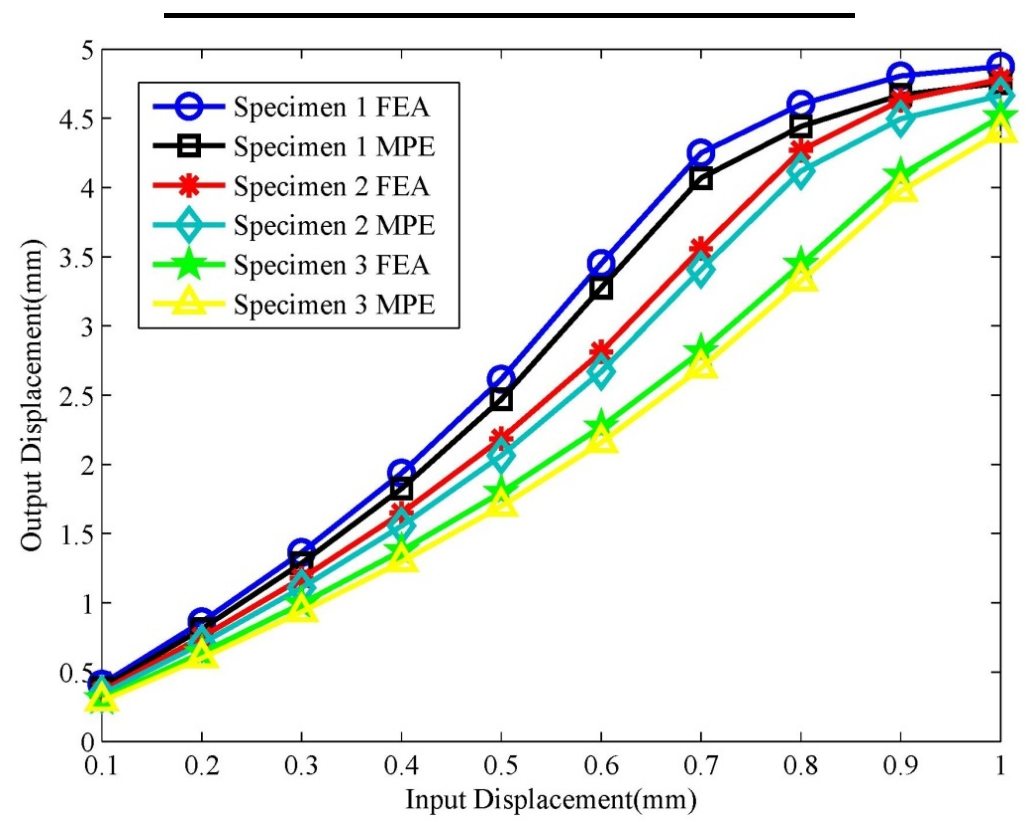

Fig. 4 The analysis results of Specimen 1, 2, 3 


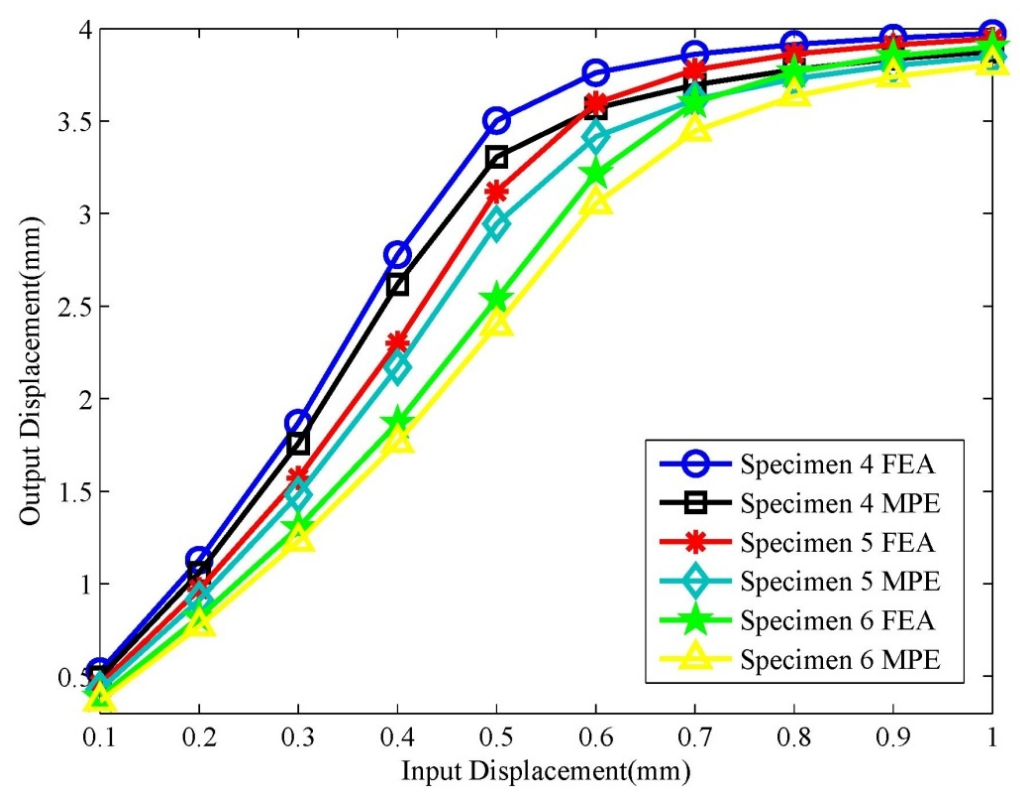

Fig. 5 The analysis results of Specimen 4, 5, 6

The analysis results are shown in Fig. 4 and Fig. 5 with that by FEA. Take the FEA as the benchmark, the MPE has an error that is less than 3\%, which verify the feasibility and precision of the MPE method.

\section{Summary:}

This article presents the MPE method for the large deformation analysis for flexure hinged displacement amplifier. The most used method for nonlinear analysis nowadays, FEA, is time-consuming and complicated. But the MPE method is accessible. And compared with FEA, results by MPE has a max error of $3 \%$.

\section{References}

[1] A P Boresi, Sidebottom O M. Advanced Mechanics of Materials. John Wiley and Sons, 1985: 763

[2] L L Howell . Compliant Mechanisms. John Wiley \& Sons, New York, 2001

[3] B V S N Reddy, S V Naik, A Saxena. Systematic Synthesis of Large Displacement Contact-Aided Monolithic Compliant Mechanisms. J Mec Des, 134(1) (2011)

[4] G B Hao, X W Kong, R L Reuben. A nonlinear analysis of spatial compliant parallel modules: Multi-beam modules. Mech Mach Theory, 46(5) (2011) 680-706

[5] Q T Aten, B D Jensen, L L Howell. Geometrically non-linear analysis of thin-film compliant MEMS via shell and solid elements. Finite Elem Anal Des, 49(1) (2012) 70-77 